\section{Le traitement du gliome par les agents chimiothérapeutiques favorise la survie des cellules souches tumorales}

Anne-Marie Bleau, Eric C. Holland
E.C. Holland : Department of Cancer Biology and Genetics, Memorial Sloan-Kettering Cancer Center,

New York 10065, États-Unis.

Brain Tumor Center, Memorial Sloan-Kettering Cancer Center,

New York 10065, États-Unis.

Department of Neurosurgery and Surgery,

Memorial Sloan-Kettering Cancer Center,

New York 10065, États-Unis.

A.M. Bleau : Department of Cancer Biology and Genetics, Memorial Sloan-Kettering Cancer Center, 1275 York Avenue, New York, Ny 10065, États-Unis.

Brain Tumor Center, Memorial Sloan-Kettering Cancer Center, New York 10065, États-Unis.

bleaua@mskcc.org repose aujourd'hui sur l'exérèse chirurgicale et la radiothérapie combinées à la chimiothérapie par le témozolomide (agent alkylant). Malheureusement, ces tumeurs sont extrêmement résistantes aux traitements et la durée médiane de survie excède rarement deux ans. De plus, la croissance rapide des cellules de glioblastomes et leur pouvoir invasif ont grandement limité la mise au point de nouveaux traitements efficaces. Nos travaux dans un modèle murin de glioblastome montrent que le témozolomide augmente l'agressivité de cellules tumorales survivantes et favorise de ce fait la récurrence de la tumeur [1]. Ce phé- tance de cellules souct cancéreuses (CSC) logées au sein de la tumeur [2].

nomène s'expliquerait en partie par la chimiorésis-

\section{Exclusion du colorant Hoechst par} les cellules tumorales de glioblastomes Afin d'étudier ce processus de résistance des CSC aux agents chimiothérapeutiques, nous avons opté pour la culture de neurosphères préparées à partir de gliomes murins induits par la surexpression du gène Pdgf (platelet-derived growth factor). Cette stratégie permet de s'abstraire des cellules endothéliales cérébrales, dont une proportion importante exprime in vivo un phénotype SP (voir ci-dessous) et de ne considérer que les cellules souches tumorales issues de gliomes. Les neurosphères sont des clones de cellules dérivés des cellules souches neurales et proliférant en suspension sous forme d'agrégats. Si les cellules souches sont rares dans un cerveau normal, dans les glioblastomes, en revanche, on dénombre un nombre important de cellules tumorales capables d'engendrer la formation de neurosphères. L'incubation de ces cellules de neurosphères issues de gliomes avec le colorant Hoechst 33342 a révélé qu'une proportion élevée (10 à $20 \%$ ) de ces cellules, analysées dans des conditions particulières d'émission de fluorescence, excluaient le colorant

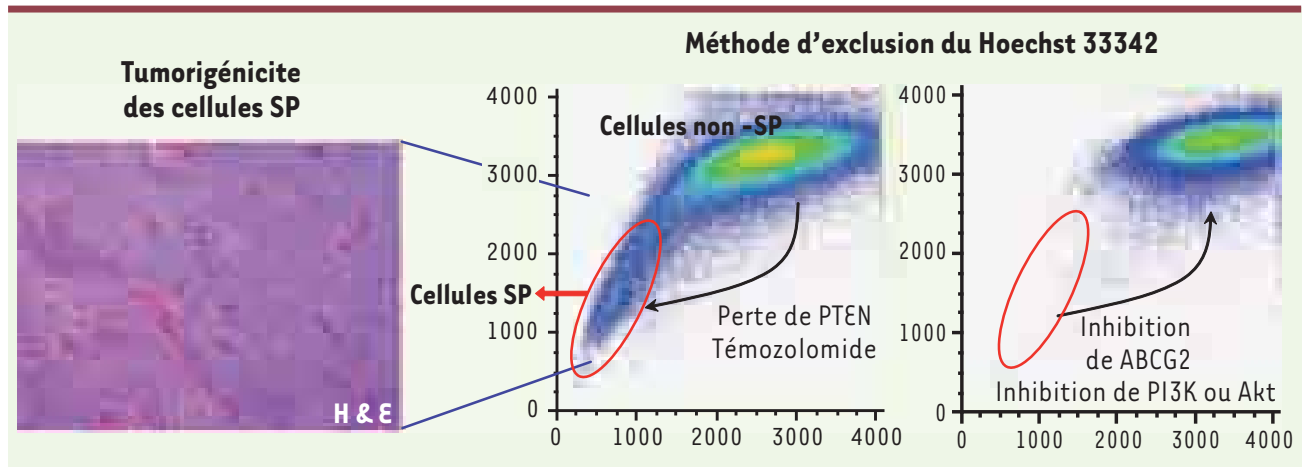

Figure 1. Efflux du colorant Hoechst 33342 par les cellules souches cancéreuses. Une propriété particulière des CSC réside dans la surexpression de protéines appartenant à la famille des transporteurs $A B C$ (transporteurs à ATP binding cassette) $[3,4]$. Ces pompes transmembranaires participent à la résistance aux médicaments par le transport actif des agents chimiothérapeutiques au travers de la membrane plasmique [5]. Le colorant fluorescent Hoechst 33342 est également un substrat des transporteurs ABC dont l'activité permet l'identification des CSC par cytométrie en flux : Ia majorité des cellules tumorales accumulent le colorant Hoechst tandis que les CSC l'expulsent par leurs transporteurs ABC [6]. Panneau du milieu. Les cellules peu fluorescentes sont appelées cellules SP (side population). La coloration au Hoechst montre que les cellules cancéreuses isolées à partir de gliomes murins possèdent un nombre élevé de cellules non fluorescentes SP, se projetant dans le cercle rouge. Cette population de cellules SP augmente à la suite de la délétion du gène PTEN ou après une incubation prolongée avec le médicament témozolomide. Panneau de droite. À l'opposé, l'inhibition de l'activité du transporteur ABCG2 ou de la voie PI3/Akt réduit le nombre de cellules SP. Un exemple d'analyse histologique de tumeur du cerveau induite par l'injection de cellules SP et colorée par hématoxyline-éosine (H \& E) est montré sur le panneau de gauche. 


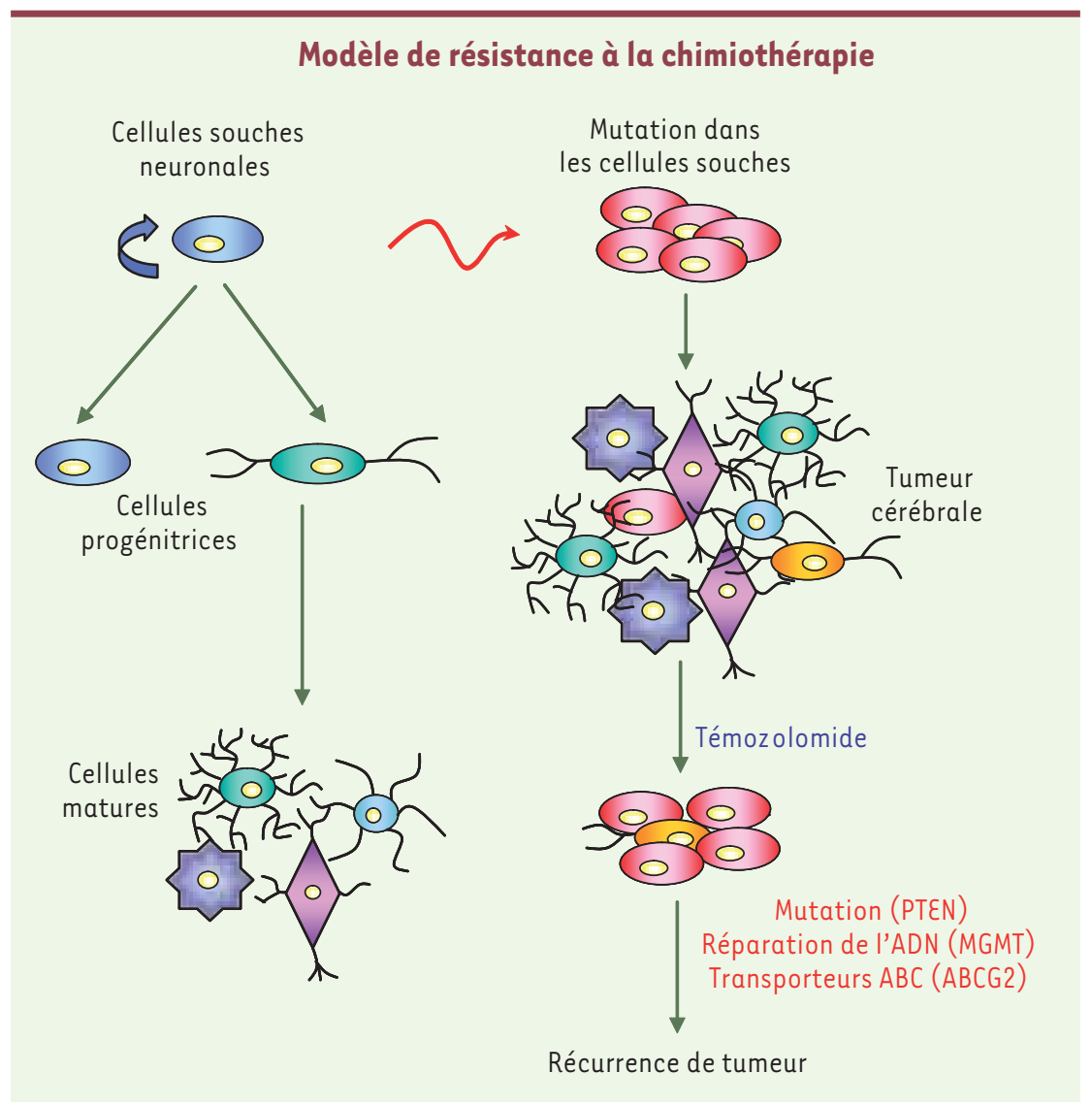

et correspondaient à la population dite SP (side population) (voir Figure I pour une explication de la technique). Nous avons montré que comparativement aux cellules non SP, les cellules SP des neurosphères tumorales sont dotées: (1) d'une prédisposition accrue à former des neurosphères secondaires, témoignant d'un haut degré d'autorenouvellement, (2) d'une plus grande résistance à l'agent antinéoplasique mitoxantrone (anthracenedione), un substrat spécifique du transporteur ABCG2 (ATP-binding cassette, sub-family $G$, member 2 ). Ce transporteur est impliqué dans l'activité MDR (multidrug resistance) par efflux des molécules cytotoxiques et dans l'exclusion du colorant Hoechst; et (3) d'un pouvoir tumorigène supérieur (évalué après l'injection des cellules dans le cerveau de souris) (Figure 1, panneau de gauche). Ces données sont compatibles avec un rôle dans le processus tumoral du transporteur ABCG2 normalement exprimé par les cellules souches héma- topoïétiques et neuronales (Figure l, panneau de droite).

Nous avons donc évalué le potentiel oncogénique de $A B C G 2$ en introduisant dans le cerveau de souriceaux nouveaunés des cellules surexprimant le gène Abcg2. Aucune tumeur n'a été détectée jusqu'à 6 mois après l'injection. De plus, nous avons montré que la coinjection de cellules exprimant Abcg2 n'augmente pas le potentiel tumorigène des cellules exprimant le gène $P d g f$. Ces résultats démontrent que le transporteur ABCG2 per se n'est pas tumorigène ; il représenterait plutôt un marqueur indépendant des CSC de gliomes, via sa fonction d'exclusion du Hoechst.

\section{Influence de la perte de PTEN} sur l'expression des cellules excluant le Hœchst

La perte du gène suppresseur de tumeur Pten (phosphatase and tensin homo$\log$ ) est une des altérations génomiques les plus fréquemment observées
Figure 2. Modèle de résistance des cellules souches cancéreuses au médicament témozolomide. L'accumulation d'altérations génomiques dans les cellules souches normales serait à l'origine du développement de tumeurs du cerveau. Grâce à leur capacité à réparer les dommages causés à l'ADN, à l'activation des voies de survie et à la surexpression du transporteur $A B C G 2$, les cellules souches cancéreuses sont responsables de la récidive des gliomes.

durant la progression des glioblastomes. Afin d'étudier les conséquences de cette altération, nous avons créé une délétion spécifique du gène Pten dans notre modèle murin de gliome grâce au système Cre-loxp. Notre équipe a été la première à montrer qu'une des conséquences de la perte de PTEN est le doublement du nombre de cellules SP dans les neurosphères issues de la prolifération des cellules de gliomes n'exprimant plus Pten et l'augmentation de la résistance des tumeurs au mitoxantrone. PTEN est un antagoniste de la PI3K parce qu'il retire le phosphate en $3^{\prime}$ de PIP3 et atténue de ce fait le signal en aval de PI3K. On sait que l'invalidation de Pten entraîne une activation de la voie Akt, et de fait, l'augmentation des cellules de phénotype SP est annulée par l'inhibition de l'activité des kinases PI3K et Akt, mais pas par celle de mTOR (target of rapamycine) située en aval de ces voies (Figure 1, panneau de droite). D'autre part, l'inhibiteur de Akt entraîne la délocalisation de ABCG2 de la membrane vers le cytoplasme, ce qui peut interférer avec l'activité du transporteur.

\section{Augmentation de la proportion} de cellules SP par le témozolomide Dans le traitement standard du glioblastome par chimiothérapie, les patients reçoivent habituellement le témozolomide durant 6 mois; on interrompt toutefois ce traitement si la tumeur continue de progresser. La récidive tumorale nous a conduits à évaluer l'effet du témozolomide sur le phénotype SP 
(CSC peu fluorescentes) en nous fondant sur l'hypothèse selon laquelle les CSC possèdent une résistance accrue au traitement, ce qui entraîne la sélection de cette population. Des neurosphères établies à partir de gliomes murins induits par le PDGF ont été exposées pendant 2 semaines à des doses croissantes de témozolomide; ce traitement a provoqué une augmentation significative $(x 1,5)$ de la proportion de cellules SP (30\%). D'autre part, la délétion du gène Pten dans ce modèle a un effet additif sur l'augmentation de la proportion de cellules SP au sein des neurosphères, qui peut parfois atteindre $75 \%$. Ce résultat indique que la perte de la protéine PTEN pourrait jouer un rôle défavorable dans la réponse au traitement chez les patients recevant du témozolomide. Qui plus est, le traitement par le témozolomide augmente le caractère tumorigène des cellules SP.

Contrairement à ce qui était anticipé, le témozolomide n'est pas un substrat pour $A B C G 2$, et il n'augmente la quantité ni de transcrits ni de protéine de ce transporteur, excluant que l'activité accrue de ce transporteur soit la cause de la sélection des CSC. En revanche, les cellules SP surexpriment la 06-méthylguanine ADN méthyltransférase (MGMT), enzyme clé dans le mécanisme de résistance des glioblastomes aux agents alkylants, puisqu'elle retire les groupements alkyl induits par ces derniers. Cette observation confirme la tendance générale des CSC à surexprimer des gènes engagés dans la réparation de I'ADN. La Figure 2 présente un modèle général décrivant les mécanismes de résistance des CSC au témozolomide.

\section{Implications thérapeutiques}

En conclusion, nous croyons qu'une double thérapie cherchant à éradiquer non seulement les cellules tumorales mais aussi les cellules souches cancéreuses serait un progrès considérable dans le traitement de patients souffrant d'un glioblastome. Nos résultats suggèrent qu'un blocage de la voie Akt diminue l'activité des transporteurs $A B C$ dans les cellules souches cancéreuses comme au niveau de la barrière hémato-encéphalique, favorisant ainsi une meilleure pénétration des médicaments dans le cerveau et l'éradication de la tumeur. $\diamond$

\section{NOUVELLE}

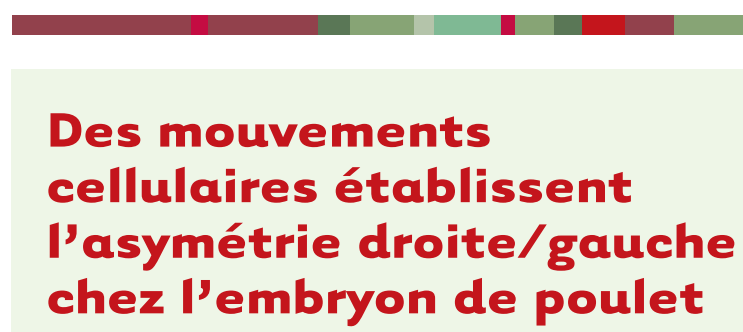

Jérôme Gros
Chimotherapeutic treatment of gliomas increases the amount of cancer stem-like cells

\section{CONFLIT D'INTÉRÊTS}

Les auteurs déclarent n'avoir aucun conflit d'intérêts concernant les données publiées dans cet article.

\section{RÉFÉRENCES}

1. Bleau AM, Hambardzumyan D, Ozawa T, et al. PTEN/ $\mathrm{PI} 3 \mathrm{~K} / \mathrm{Akt}$ pathway regulates the side population phenotype and ABCG2 activity in glioma tumor stem-like cells. Cell Stem Cell 2009 ; 4 : 226-35.

2. Regenbrecht CR, Lehrach H, Adjaye J. Stemming cancer: functional genomics of cancer stem cells in solid tumors. Stem Cell Rev 2008 ; 4 : 319-28.

3. Bao S, Wu Q, McLendon RE, et al. Glioma stem cells promote radioresistance by preferential activation of the DNA damage response. Nature 2006 ; 444 : 756-60.

4. Dean M, Fojo T, Bates S. Tumour stem cells and drug resistance. Nat Rev Cancer 2005 ; 5 : 275-84.

5. Hirschmann-Jax C, Foster AE, Wulf GG, et al. A distinct side population of cells with high drug efflux capacity in human tumor cells. Proc Natl Acad Sci USA 2004 ; $101: 14228-33$.

6. Goodell MA, Brose K, Paradis G, et al. Isolation and functional properties of murine hematopoietic stem cells that are replicating in vivo. J Exp Med 1996 ; 183: 1797-806.

7. Shih AH, Dai C, Hu X, et al. Dose-dependent effects of platelet-derived growth factor-B on glial tumorigenesis. Cancer Res 2004 ; 64 : 4783-9.

8. McConville P, Hambardzumyan D, Moody JB, et al. Magnetic resonance imaging determination of tumor grade and early response to temozolomide in a genetically engineered mouse model of glioma. Clin Cancer Res 2007 ; 13 : 2897-904.
Department of Genetics, Harvard Medical School, Boston MA 02115, États-Unis. jgros@genetics.med.harvard.edu
> Les vertébrés affichent extérieurement une symétrie bilatérale presque parfaite. En revanche, la forme et la position des organes à l'intérieur du corps sont asymétriques: chez l'homme, le cœur est incliné à gauche du corps, le foie est positionné en majeure partie à droite, le poumon gauche comporte deux lobes tandis que le droit en comporte trois, etc. Cette asymétrie droite/gauche $(D / G)$ dans l'organisation des organes est à la base de leur fonctionnement au sein de la cavité interne du corps. L'asymétrie $D / G$ se met en place très tôt au cours de l'embryogenèse. Le premier signe moléculaire d'asymétrie, décrit chez tous les vertébrés étudiés à ce jour (souris, poulet, lapin, porc, grenouille), est détecté bien avant la formation des organes, au moment de la gastrulation lorsque l'expression du gène nodal apparaît exclusivement dans la partie gauche de l'embryon. Cette expression asymétrique de Nodal engendre l'acquisition d'une identité de côté gauche en opposition à une identité de côté droit (où nodal n'est pas exprimé). 